\title{
A Characterization of the Distributions That Imply Existence of Linear Equilibria in the Kyle-Model*
}

\section{Georg Nöldeke and Thomas Tröger}

Department of Economics, University of Bonn, Adenauerallee 24-42, 53113 Bonn, Germany

(e-mail: georg.noldeke@uni-bonn.de and ttroeger@uni-bonn.de)

\section{Summary.}

The existence of a linear equilibrium in Kyle's model of market making with multiple, symmetrically informed strategic traders is implied for any number of strategic traders if the joint distribution of the underlying exogenous random variables is elliptical. The reverse implication has been shown for the case in which the random variables are independent and have finite second moments. Here we extend this result to the case in which the underlying random variables are not necessarily independent and their joint distribution is determined by its moments.

Keywords and Phrases: Market Microstructure, Kyle Model, Linear Equilibria, Elliptical Distributions

JEL Classification Numbers: G14, D82

*Financial Support by the Deutsche Forschungsgemeinschaft through SFB/TR 15 is gratefully acknowledged. 


\section{INTRODUCTION}

Most models in the market microstructure literature (see [16] for a survey) consider linear equilibria. As argued by Bagnoli, Viswanathan and Holden [1] it is thus important to understand the conditions under which such linear equilibria exist. The purpose of this paper is to contribute to this task in the context of a Kyle-type model [12] with multiple, symmetrically informed strategic traders. In our model there are $N$ risk-neutral strategic traders who observe the payoff of a risky asset before submitting market orders. In addition there are exogenous market orders (noise trade). Risk-neutral market makers observe the aggregate net order flow, choose prices and then execute every order. Throughout the paper we assume that the exogenous random variables - asset payoff and noise trade - have finite second moments.

Under the additional assumption that asset payoff and noise trade are independent Bagnoli, Viswanathan and Holden [1] show that in this model a linear equilibrium exists for any number $N$ of strategic traders if and only if the joint distribution of the exogenous random variables is normal. ${ }^{1}$

We ask whether a similar characterization can be obtained without imposing the independence assumption. To motivate this question we observe, first, that Foster and Viswanathan [9] have shown that relaxing the independence assumption is essential in generating phenomena such as the dependence of price volatility and trading volume on the arrival of news in the context of a Kyle-type model. Second, while it may seem natural to assume that "pure" noise trading and asset payoff are independent, in the context of the Kyle-model noise trading should be interpreted as the aggregate market order of all traders whose strategic behavior is not explicitly taken into account. From this perspective we see no compelling justification why noise trade and payoff must be independent. ${ }^{2}$

Foster and Viswanathan [9] show that a linear equilibrium exists for any number of strategic traders if the joint distribution of the exogenous random variables is elliptical. ${ }^{3}$ For the case in which asset payoff and noise trade are

\footnotetext{
${ }^{1}[1]$ derive this result under an additional technical condition on the characteristic function of the random variables. Nöldeke and Tröger [14] show that this condition can be dispensed with and that normality is already implied by requiring the existence of linear equilibria for two different numbers of strategic traders.

${ }^{2}$ Suppose, for instance, that noise trading is viewed as arising from liquidity shocks. While it seems reasonable to suppose that such liquidity shocks have an idiosyncratic component, it seems equally reasonable to suppose that they also have a component determined by economic fundamentals also affecting the payoff of the risky asset, invalidating the independence assumption.

${ }^{3} \mathrm{~A}$ multivariate random variable has an elliptical distribution if it is an affine trans-
} 
independent the result from Bagnoli, Viswanathan and Holden [1] implies the reverse (because normal distributions are the only elliptical distributions satisfying the independence condition), i.e. the ellipticity condition is tight for the independent case. Here we show that the same is true more generally, that is without assuming independence of asset payoff and noise trade, provided the additional condition is satisfied that the joint distribution of the exogenous random variables is determined by its moments. The spirit of this result is similar to Chamberlain [6] who obtains a characterization of elliptical distributions as those that imply mean-variance preferences in a standard portfolio problem.

The remainder of this paper is organized as follows. Section 2 presents the extension of the Kyle-model to the case of $N$ strategic traders. Section 3 introduces the definition of linear equilibrium that we employ in this paper and provides a preliminary necessary and sufficient condition for their existence. Section 4 establishes our characterization of elliptical distributions by connecting the necessary and sufficient condition from the previous section to the rotation invariance of the distribution of the standardized exogenous random variables. Section 5 concludes. Proofs are in the Appendix.

\section{The Model}

The model extends the single auction setting considered in Kyle ([12], Section 2) by allowing for multiple strategic traders as in [1] and [14]. There are three types of traders: noise traders, risk neutral market makers, and $N \geq 1$ risk neutral strategic traders. The aggregate quantity traded by noise traders and the payoff of the risky asset are given by exogenous random variables. Noise trading is denoted by $\tilde{u}$. The payoff of the risky asset is denoted by $\tilde{v}$.

We do not assume that $\tilde{u}$ and $\tilde{v}$ are independent, but maintain the assumption that noise trade and asset payoff are uncorrelated. ${ }^{4}$ In addition, we assume that these random variables have strictly positive, finite second moments. ${ }^{5}$

formation of a random variable with a rotation invariant distribution. See Section 4 for formal definitions and [8] for a detailed exposition. [9] presents many examples of elliptical distributions and provides references to applications in financial economics.

${ }^{4}$ This assumption is made to ease comparison with the existing literature. As we argue in Appendix B our results do not require it.

${ }^{5}$ The assumption that second moments are finite is necessary for a linear equilibrium as defined below to exist - otherwise the expected payoff of a strategic trader would be infinite. 
Assumption 1 The distribution of $(\tilde{u}, \tilde{v})$ has finite second moments satisfying $\sigma_{u}^{2}=\operatorname{Var}[\tilde{u}]>0, \sigma_{v}^{2}=\operatorname{Var}[\tilde{v}]>0$, and $\operatorname{Cov}[\tilde{u}, \tilde{v}]=0$.

All strategic traders observe the realization of $\tilde{v}$, but not of $\tilde{u}$, and then simultaneously decide on the market order they submit. A strategy for the strategic trader $n=1, \cdots, N$ is given by a Lebesgue measurable function $X_{n}: \mathbb{R} \rightarrow \mathbb{R}$, determining his market order as a function of the observed payoff. For a given strategy $X_{n}$, let $\tilde{x}_{n}=X_{n}(\tilde{v})$. A strategy combination $\left(X_{1}, \cdots, X_{N}\right)$ determines the order flow as $\tilde{y}=\sum_{n} \tilde{x}_{n}+\tilde{u}$.

Market makers observe the realization of the order flow, but not any of its components, and engage in a competitive auction to serve the order flow. The outcome of this competition is described by a Lebesgue measurable function $P: \mathbb{R} \rightarrow \mathbb{R}$, called the pricing rule. Given $\left(P, X_{1}, \cdots, X_{N}\right)$ define $\tilde{p}=P(\tilde{y})$ and let $\tilde{\pi}_{n}=(\tilde{v}-\tilde{p}) \tilde{x}_{n}$ denote the resulting profit of trader $n$. To ensure that the expected profit of a strategic trader is well-defined for all feasible $\left(P, X_{1}, \cdots, X_{N}\right)$, we restrict the strategy set of a strategic trader to $\mathcal{X}=\left\{X_{n}: \mathbb{R} \rightarrow \mathbb{R} \mid E\left[\tilde{x}_{n}^{2}\right]<\infty\right\}$ and the set of pricing rules to $\mathcal{P}=\left\{P \mid \forall\left(X_{1}, \cdots, X_{N}\right) \in \mathcal{X}^{N}: E\left[\tilde{p}^{2}\right]<\infty\right\}$.

The equilibrium conditions are that the competition between market makers drives their expected profits to zero conditional on the order flow and that each strategic trader chooses his trading strategy to maximize his expected profits.

Definition $1\left(P, X_{1}, \cdots, X_{N}\right) \in \mathcal{P} \times \mathcal{X}^{N}$ is an equilibrium if

$$
E[\tilde{v}-\tilde{p} \mid \tilde{y}]=0
$$

and, for all $n$ and $X \in \mathcal{X}$,

$$
E\left[\tilde{\pi}_{n}\right] \geq E\left[\left(\tilde{v}-P\left(\sum_{m \neq n} \tilde{x}_{m}+X(\tilde{v})+\tilde{u}\right)\right) X(\tilde{v})\right] .
$$

\section{Linear EqUiLibria}

The convention in the literature is to call an equilibrium linear if the pricing rule is an affine function. In the following definition we add a linearity requirement on the aggregate market order of the strategic traders. Given the motivation for the study of linear equilibria, namely to obtain simple closed-form expressions for equilibria, we find it natural to focus on equilibria satisfying this additional linearity requirement. When the exogenous 
random variables are independent this additional requirement is redundant because it is implied by linearity of the pricing rule. ${ }^{6}$

Definition 2 An equilibrium $\left(P, X_{1}, \cdots, X_{N}\right)$ is linear if there exist $(\mu, \lambda) \in$ $\mathbb{R}^{2}$ such that

$$
\forall y: P(y)=\mu+\lambda y
$$

and $(\alpha, \beta) \in \mathbb{R}^{2}$ such that

$$
\sum_{n} \tilde{x}_{n}=\alpha+\beta \tilde{v}
$$

For the case in which $\tilde{u}$ and $\tilde{v}$ are independent [1] have shown that the parameters describing a linear equilibrium (if it exists) are uniquely determined and depend only on the number of strategic traders and the first two moments of the distribution. We begin our analysis by showing that the same characterization applies under Assumption 1. Towards this end let

$$
\lambda_{N}=\frac{\sqrt{N}}{N+1} \frac{\sigma_{v}}{\sigma_{u}}, \quad \mu_{N}=E[\tilde{v}]-\lambda_{N} E[\tilde{u}]
$$

and

$$
\alpha_{N}=-\sqrt{N} \frac{\sigma_{u}}{\sigma_{v}} E[\tilde{v}], \quad \beta_{N}=\sqrt{N} \frac{\sigma_{u}}{\sigma_{v}} .
$$

Proposition 1 If $\left(P, X_{1}, \cdots X_{N}\right)$ is a linear equilibrium then

$$
\tilde{y}=\tilde{y}_{N} \equiv \alpha_{N}+\beta_{N} \tilde{v}+\tilde{u}
$$

and

$$
\tilde{p}=\tilde{p}_{N} \equiv \mu_{N}+\lambda_{N} \tilde{y}_{N}
$$

The proof of Proposition 1 begins by assuming a linear pricing rule and solving for the aggregate market order of the strategic traders implied by (2). The linearity requirement (4) then implies $E[\tilde{u} \mid \tilde{v}]=E[\tilde{u}]$ and we obtain the order flow $\tilde{y}$ and the price $\tilde{p}$ as affine functions of the exogenous random variables. The argument is completed by noting that due to the market efficiency condition (1) every equilibrium must satisfy the condition that

\footnotetext{
${ }^{6}$ More generally, it is easy to see (cf. the proof of Proposition 1 ) that the additional requirement is redundant if $\tilde{u}$ and $\tilde{v}$ are not only uncorrelated but satisfy $E[\tilde{u} \mid \tilde{v}]=E[\tilde{u}]$. This is similar to the sufficient conditions for the existence of a linear equilibrium imposed by [1] in the context of a Kyle-type model with differentially informed strategic traders. It remains an open question whether equilibria with a linear pricing rule exist if $\tilde{u}$ and $\tilde{v}$ are uncorrelated and the condition $E[\tilde{u} \mid \tilde{v}]=E[\tilde{u}]$ is violated.
} 
the forecast error $\tilde{v}-\tilde{p}$ has zero expectation and is uncorrelated with the order flow $\tilde{y}$. This provides two equations that can be used to verify that $\lambda=\lambda_{N}$ and $\mu=\mu_{N}$, hence (8). Substituting the expressions from (5) into the order flow yields (7).

This proof makes clear that to characterize linear equilibria the equilibrium condition (1) is not fully exploited. The proof only uses that the forecast error $\tilde{v}-\tilde{p}$ has expectation 0 and is uncorrelated with the order flow. To find a necessary condition for the existence of a linear equilibrium for all $N$ we fully exploit (1), namely that the expectation of the forecast error equals 0 conditional on the observation of the order flow; i.e.,

$$
E\left[\tilde{v}-\tilde{p}_{N} \mid \tilde{y}_{N}\right]=0 \quad \forall N \in \mathbb{N} .
$$

This provides a condition on the joint distribution of $(\tilde{u}, \tilde{v})$. It will be convenient to write this condition in terms of standardized random variables. Let

$$
\tilde{u}^{*}=\frac{\tilde{u}-E[\tilde{u}]}{\sigma_{u}}, \quad \tilde{v}^{*}=\frac{\tilde{v}-E[\tilde{v}]}{\sigma_{v}}
$$

denote the standardization of the random variables $(\tilde{u}, \tilde{v})$. Straightforward algebra shows that (9) is equivalent to

$$
E\left[-\frac{\sqrt{N}}{\sqrt{N+1}} \tilde{u}^{*}+\frac{1}{\sqrt{N+1}} \tilde{v}^{*} \mid \frac{1}{\sqrt{N+1}} \tilde{u}^{*}+\frac{\sqrt{N}}{\sqrt{N+1}} \tilde{v}^{*}\right]=0 \quad \forall N \in \mathbb{N} .
$$

It should be clear from the above discussion that (10) is necessary for the existence of a linear equilibrium for all $N$. The proof of the following proposition shows that (10) is also sufficient. Establishing this is more subtle because it involves the demonstration that (4) is implied if (10) holds.

Proposition $2 A$ linear equilibrium exists for all $N \in \mathbb{N}$ if and only if (10) holds.

The characterization result in Proposition 2 is identical to the one we have established for the case of independent $\tilde{u}$ and $\tilde{v}$ in our previous paper [14]. In [14] we also show that under the independence assumption condition (10) is equivalent to normality of the joint distribution of $(\tilde{u}, \tilde{v})$. It is clear that this characterization fails without the independence assumption because it is known (cf. [9]) that the ellipticity of the joint distribution of $(\tilde{u}, \tilde{v})$ is sufficient for the existence of linear equilibria for all $N$. In the following we thus investigate the question whether ellipticity of the distribution of $(\tilde{u}, \tilde{v})$ is also necessary for (10). 


\section{Rotation InVARIAnCe AND Ellipticity}

For all $\eta \in(0,2 \pi)$, let $\left(\tilde{u}_{\eta}^{*}, \tilde{v}_{\eta}^{*}\right)$ denote the bivariate random variable obtained from a rotation of $\left(\tilde{u}^{*}, \tilde{v}^{*}\right)$ by the angle $\eta$ around the origin, i.e.

$$
\left(\tilde{u}_{\eta}^{*}, \tilde{v}_{\eta}^{*}\right)=\left(\cos \eta \tilde{u}^{*}+\sin \eta \tilde{v}^{*},-\sin \eta \tilde{u}^{*}+\cos \eta \tilde{v}^{*}\right) .
$$

Definition 3 (Cf. [8]) The distribution of $(\tilde{u}, \tilde{v})$ satisfying Assumption 1 is elliptical if the distribution of $\left(\tilde{u}^{*}, \tilde{v}^{*}\right)$ is rotation invariant. The distribution of $\left(\tilde{u}^{*}, \tilde{v}^{*}\right)$ is rotation invariant if for all $\eta \in(0,2 \pi)$ the random variable $\left(\tilde{u}_{\eta}^{*}, \tilde{v}_{\eta}^{*}\right)$ has the same distribution as $\left(\tilde{u}^{*}, \tilde{v}^{*}\right)$.

Rewriting (10) as

$$
E\left[\tilde{v}_{\eta}^{*} \mid \tilde{u}_{\eta}^{*}\right]=0 \quad \forall \eta \in A,
$$

where

$$
A=\left\{\eta \in(0,2 \pi) \mid \exists N \in \mathbb{N}: \frac{\sqrt{N}}{\sqrt{N+1}}=\sin \eta\right\},
$$

suggests that (10) is closely related to the condition that $\left(\tilde{u}^{*}, \tilde{v}^{*}\right)$ is rotation invariant. Indeed, if one replaces the set $A$ in (12) by the full set of angles $(0,2 \pi)$ then one obtains a condition that is equivalent to the rotation invariance of $\left(\tilde{u}^{*}, \tilde{v}^{*}\right)$ and thus the ellipticity of $(\tilde{u}, \tilde{v})$.

Remark 1 The distribution of $(\tilde{u}, \tilde{v})$ is elliptical if and only if

$$
E\left[\tilde{v}_{\eta}^{*} \mid \tilde{u}_{\eta}^{*}\right]=0, \forall \eta \in(0,2 \pi) .
$$

The proof is similar to an argument used in Hardin's [11] characterization of distributions satisfying the linear regression property. It shows that (13) is equivalent to the condition that the derivative of the characteristic function of $\left(\tilde{u}^{*}, \tilde{v}^{*}\right)$ with respect to the polar angle equals zero, implying rotation invariance.

Because condition (13) implies (12), the result that a linear equilibrium exists for all $N \in \mathbb{N}$ if the distribution of $(\tilde{u}, \tilde{v})$ is elliptical is an immediate consequence of Proposition 2 and Remark 1. The difficulty with establishing the reverse conclusion is that the set $A$ appearing in condition (12) only contains isolated points with a single limit point. I.e., condition (12) provides much less information than (13). More precisely, the condition that the derivative of the characteristic function of $\left(\tilde{u}^{*}, \tilde{v}^{*}\right)$ with respect to the polar angle equals zero is implied by (12) only for a countable set of isolated lines 
through the origin, precluding the possibility to obtain the rotation invariance of the characteristic function (and hence the distribution) of $\left(\tilde{u}^{*}, \tilde{v}^{*}\right)$ from (12) alone. As we show in the proof of the following proposition the conditions are, however, sufficiently rich to demonstrate the rotation invariance of the partial derivatives of the characteristic function in the origin. Assuming that all moments of the distribution of $\left(\tilde{u}^{*}, \tilde{v}^{*}\right)$ are finite, these partial derivatives determine the moments of the distribution, and we can conclude that the moments are rotation invariant. ${ }^{7}$

Proposition 3 Suppose condition (12) holds and all moments of $\left(\tilde{u}^{*}, \tilde{v}^{*}\right)$ are finite. Then the moments of $\left(\tilde{u}^{*}, \tilde{v}^{*}\right)$ are rotation invariant, that is for all $\eta \in(0,2 \pi)$ the random variable $\left(\tilde{u}_{\eta}^{*}, \tilde{v}_{\eta}^{*}\right)$ has the same moments as $\left(\tilde{u}^{*}, \tilde{v}^{*}\right)$.

It is clear that if the distribution of $\left(\tilde{u}^{*}, \tilde{v}^{*}\right)$ is determined by its moments, i.e. if all moments of the distribution are finite and there exists no other distribution with the same moments, then rotation invariance of the moments implies rotation invariance of the distribution. Because the distribution of $(\tilde{u}, \tilde{v})$ is determined by its moments if and only if the same is true for the distribution of $\left(\tilde{u}^{*}, \tilde{v}^{*}\right)$ we have thus established:

Theorem 1 Suppose the joint distribution of $(\tilde{u}, \tilde{v})$ is determined by its moments and a linear equilibrium exists for all $N \in \mathbb{N}$. Then the distribution of $(\tilde{u}, \tilde{v})$ is elliptical.

We note that the condition that the joint distribution of $\left(\tilde{u}^{*}, \tilde{v}^{*}\right)$ is determined by its moments is indispensable to obtain Theorem 1 from Proposition 3: given any rotation invariant distribution that is not determined by its moments, one can construct a distribution with the same moments that is not rotation invariant. ${ }^{8}$ Hence, to extend Theorem 1 to a larger class of

\footnotetext{
${ }^{7}$ The general idea of deriving properties of the moments from properties of the characteristic function along a countable set of lines through the origin already appears in Gilbert [10], albeit in a different context. Gilbert shows that the moments of a two-dimensional distribution are determined by the moments of any infinite set of one-dimensional projections of the distribution.

8 Any rotation invariant distribution $F$ can be decomposed into two independent distributions: the uniform distribution over directions in the plane, and a distribution $G$ over Euclidian distances from the origin. In this decomposition any distribution over Euclidian distances can be used, so that there is a one-to-one correspondence between distributions $G$ on $[0, \infty)$ and the set of rotation invariant distributions $F$. Berg and Thill [3] show that $G$ is determined by its moments only if $F$ is. Hence, assuming that $F$ is not determined by its moments, there exists a distribution $G^{\prime}$ over Euclidian distances from the origin such that $G^{\prime} \neq G$ and $G^{\prime}$ has the same moments as $G$. Define a distribution $F^{\prime}$ by the
} 
elliptical distributions one would need to use implications of the equilibrium conditions that go beyond the rotation invariance of the moments, which appears difficult even under the assumption that all moments are finite. ${ }^{9}$ It is clear, though, that Theorem 1 applies under any primitive assumption on the distribution function which, in conjunction with the equilibrium conditions, implies that the joint distribution of $(\tilde{u}, \tilde{v})$ is determined by its moments. For instance, supposing that all moments are finite and that one of the marginal distributions is determined by its moments will do: ${ }^{10}$ from Proposition 3 it then follows that both marginal distributions are determined by their moments which suffices to conclude that the joint distribution is determined by its moments [17]. Because there exist elliptical distributions that are not determined by their moments (cf. footnote 8 ) it is also clear that such an approach will not yield a full characterization of the elliptical class. This is in contrast to the case in which $\tilde{u}$ and $\tilde{v}$ are independent, where the equilibrium conditions alone imply that the joint distribution of $(\tilde{u}, \tilde{v})$ is determined by its moments (cf. the proof of Proposition 2 in [14]).

\section{Concluding Remarks}

We have shown that existence of linear equilibria in a Kyle-type model with multiple strategic traders implies that the joint distribution of asset payoff and noise trade is elliptical if this distribution is determined by its moments. As we have explained, the assumption that the joint distribution is determined by its moments is restrictive and, unfortunately, not easy to relax. The assumption does, however, hold in many cases of interest. For instance, any distribution that has a moment generating function in a neighborhood of zero is determined by its moments; in particular, the assumption holds if the distribution has bounded support. More generally,

following sampling procedure. A direction in the plane is chosen according to the uniform distribution; if the angle falls into the interval $[0, \pi]$, a distance from the origin is chosen according to $G$; otherwise, a distance from the origin is chosen according to $G^{\prime}$. It is straightforward to verify that $F^{\prime}$ has the same moments as $F$. The distribution $F^{\prime}$ is, of course, not rotation invariant.

${ }^{9}$ Recall that the equilibrium conditions are equivalent to conditions on the characteristic function of $\left(\tilde{u}^{*}, \tilde{v}^{*}\right)$ along a countable set of isolated lines through the origin. Bélisle, Massé, and Ransford [4] show that there exist two different 2-dimensional distributions that have the same finite moments and identical characteristic functions along an infinite set of lines through the origin.

${ }^{10}$ Note that this assumption is neither implied by (not even if the joint distribution is known to be rotation invariant) nor (without rotation invariance of the moments) implies the assumption that the joint distribution is determined by its moments, cf. [2]. 
Cramér and Wold [7] show (see also Shohat and Tamarkin [19]) that the distribution of $(\tilde{u}, \tilde{v})$ is determined by its moments if the following twodimensional extension of the Carleman condition holds:

$$
\sum_{k=1}^{\infty}\left(E\left[\tilde{v}^{2 k}\right]+E\left[\tilde{u}^{2 k}\right]\right)^{-1 / 2 k}=\infty .
$$

For more recent results on the multidimensional problem of moments we refer to Berg [2].

In a previous version of this paper [15] we have shown that our main characterization result also holds in the Rochet-Vila [18] model of market making with multiple strategic traders. Because in the Rochet-Vila model the strategic traders observe the noise trade before submitting their orders, the analysis is somewhat simpler and Theorem 1 holds without imposing linearity of the strategic traders' aggregate order. 
Proof of Proposition 1: Suppose $\left(P, X_{1}, \cdots, X_{N}\right)$ is a linear equilibrium with $P(y)=\mu+\lambda y$. Because $X_{n}(v)$ is an equilibrium strategy and thus satisfies (2):

$$
X_{n}(v) \in \arg \max _{x}\left(v-\mu-\lambda\left(\sum_{m \neq n} X_{m}(v)+x+E[\tilde{u} \mid \tilde{v}=v]\right)\right) x \text { a.e. } v .
$$

Because $\tilde{v}$ is non-degenerate this condition can only be satisfied if $\lambda>0$. For $\lambda>0$ the first order condition characterizes a maximum and yields

$$
\forall n: \tilde{v}-\mu-\lambda\left(\sum_{m} \tilde{x}_{m}+\tilde{x}_{n}+E[\tilde{u} \mid \tilde{v}]\right)=0 .
$$

Hence, $\tilde{x}_{n}$ is independent of $n$ and thus

$$
\sum_{n} \tilde{x}_{n}=\frac{N(\tilde{v}-\mu-\lambda E[\tilde{u} \mid \tilde{v}])}{\lambda(N+1)} .
$$

This together with (4) implies that $E[\tilde{u} \mid \tilde{v}]$ is an affine function of $\tilde{v}$. From the assumption that $\tilde{u}$ and $\tilde{v}$ are uncorrelated, it is then straightforward that $E[\tilde{u} \mid \tilde{v}]=E[\tilde{u}]$. Consequently, (14) implies that the order flow is given by

$$
\tilde{y}=\frac{N(\tilde{v}-\mu-\lambda E[\tilde{u}])}{\lambda(N+1)}+\tilde{u}
$$

and thus

$$
\tilde{p}=\frac{N(\tilde{v}-\lambda E[\tilde{u}])+\mu}{N+1}+\lambda \tilde{u} .
$$

The market efficiency condition (1) implies

$$
E[\tilde{v}-\tilde{p}]=0
$$

and

$$
E[(\tilde{v}-\tilde{p}) \tilde{y}]=0
$$

Using (16) and (17) we obtain

$$
\mu=E[\tilde{v}]-\lambda E[\tilde{u}] .
$$

Using (19) to eliminate $\mu$ from equations (15) and (16), formula (18) together with the fact that $\tilde{u}$ and $\tilde{v}$ are uncorrelated yields $\lambda=\lambda_{N}$. Hence, $\mu=\mu_{N}$ 
follows from (19). Finally, substituting $\lambda=\lambda_{N}$ and $\mu=\mu_{N}$ into (15) yields $(7)$.

Proof of Proposition 2: To prove sufficiency of (10) define strategies $X_{n}$ and a pricing rule $P$ by

$$
X_{n}(v)=\frac{\alpha_{N}}{N}+\frac{\beta_{N}}{N} v, \quad P(y)=\mu_{N}+\lambda_{N} y .
$$

Straightforward algebra shows that (10) implies (1) for all $N$. Furthermore, the proof of Proposition 1 shows that (2) is implied by (10) if $E[\tilde{u} \mid \tilde{v}]=E[\tilde{u}]$ holds. It thus remains to show that (10) implies $E[\tilde{u} \mid \tilde{v}]=E[\tilde{u}]$.

For all $N \in \mathbb{N}$, let

$$
\tilde{w}_{N}=-\frac{\sqrt{N}}{\sqrt{N+1}} \tilde{u}^{*}+\frac{1}{\sqrt{N+1}} \tilde{v}^{*}, \quad \tilde{z}_{N}=\frac{1}{\sqrt{N+1}} \tilde{u}^{*}+\frac{\sqrt{N}}{\sqrt{N+1}} \tilde{v}^{*} .
$$

For any bounded continuous function $g: \mathbb{R} \rightarrow \mathbb{R}$ condition (10) implies $E\left[\tilde{w}_{N} g\left(\tilde{z}_{N}\right)\right]=0$ for all $N$.

The sequence of random variables $\tilde{w}_{N} g\left(\tilde{z}_{N}\right)$ converges pointwise to $-\tilde{u}^{*} g\left(\tilde{v}^{*}\right)$ and is dominated by the integrable random variable $\sup |g| \cdot\left(\left|\tilde{u}^{*}\right|+\left|\tilde{v}^{*}\right|\right)$. Hence, by Lebesgue's dominated convergence theorem ([5], Theorem 16.4), $E\left[-\tilde{u}^{*} g\left(\tilde{v}^{*}\right)\right]=0$ for any bounded continuous function $g$. Consequently, $E\left[\tilde{u}^{*} \mid \tilde{v}^{*}\right]=0$, hence $E[\tilde{u} \mid \tilde{v}]=E[\tilde{u}]$.

\section{Proof of Remark 1:}

By Theorem 6.1.1. in Lukacs and Laha [13], (13) holds if and only if

$$
\forall \eta \in(0,2 \pi), r \in \mathbb{R}: E\left[\left(-\sin \eta \tilde{u}^{*}+\cos \eta \tilde{v}^{*}\right) e^{i r\left(\cos \eta \tilde{u}^{*}+\sin \eta \tilde{v}^{*}\right)}\right]=0,
$$

or, equivalently,

$$
\begin{aligned}
& \forall \eta \in(0,2 \pi), r \in \mathbb{R}: \\
& \quad-\sin \eta \Phi_{1,0}(r \cos \eta, r \sin \eta)+\cos \eta \Phi_{0,1}(r \cos \eta, r \sin \eta)=0
\end{aligned}
$$

where $\Phi_{1,0}$ and $\Phi_{0,1}$ denote the first-order partial derivatives of the characteristic function $\Phi$ of $\left(\tilde{u}^{*}, \tilde{v}^{*}\right)$. By the chain rule, $(20)$ is equivalent to the condition that the partial derivative of $F(r, \eta)=\Phi(r \cos \eta, r \sin \eta)$ with respect to the polar angle $\eta$ is identical to zero for all $r \in \mathbb{R}$, which is equivalent to the condition that the characteristic function of $\left(\tilde{u}^{*}, \tilde{v}^{*}\right)$, and thus the distribution of $\left(\tilde{u}^{*}, \tilde{v}^{*}\right)$, is rotation invariant. 
Proof of Proposition 3: Let $\Phi$ denote the characteristic function of $\left(\tilde{u}^{*}, \tilde{v}^{*}\right)$. The characteristic function $\Phi^{\eta}$ of the rotated random vector $\left(\tilde{u}_{\eta}^{*}, \tilde{v}_{\eta}^{*}\right)$ is then given by

$$
\begin{aligned}
\Phi^{\eta}(s, t) & =E\left[e^{i s\left(\cos \eta \tilde{u}^{*}+\sin \eta \tilde{v}^{*}\right)+i t\left(-\sin \eta \tilde{u}^{*}+\cos \eta \tilde{v}^{*}\right)}\right] \\
& =\Phi(s \cos \eta-t \sin \eta, s \sin \eta+t \cos \eta) .
\end{aligned}
$$

Because all moments exist for $\left(\tilde{u}^{*}, \tilde{v}^{*}\right)$, all moments exist for $\left(\tilde{u}_{\eta}^{*}, \tilde{v}_{\eta}^{*}\right)$ and these moments are determined by the higher order partial derivatives of the characteristic functions $\Phi(s, t)$ and $\Phi^{\eta}(s, t)$ at $(0,0)$. I.e., it is sufficient to show

$$
\forall \eta \in \mathbb{R}, m, n \in \mathbb{N}_{0}: \Phi_{m, n}^{\eta}(0,0)=\Phi_{m, n}(0,0),
$$

where here (and in the following) we use notation such as $\Phi_{m, n}$ to denote (higher order) partial derivatives.

Applying Theorem 6.1.1 in [13] as in the proof of Remark 1 it follows that (12) is equivalent to

$$
\begin{aligned}
& \forall \eta \in A, r \in \mathbb{R}: \\
& \quad-\sin \eta \Phi_{1,0}(r \cos \eta, r \sin \eta)+\cos \eta \Phi_{0,1}(r \cos \eta, r \sin \eta)=0 .
\end{aligned}
$$

Substituting $\hat{s}=s \cos \eta$ and $\tan \eta=\sin \eta / \cos \eta$ (note that $\cos (\eta) \neq 0$ for all $\eta \in A$ ), we can rewrite (23) as

$$
\forall \hat{s} \in \mathbb{R}, \eta \in A: f(\hat{s}, \tan \eta \hat{s})=0,
$$

where for all $\hat{s}, \hat{t} \in \mathbb{R}$

$$
f(\hat{s}, \hat{t})=-\hat{t} \Phi_{1,0}(\hat{s}, \hat{t})+\hat{s} \Phi_{0,1}(\hat{s}, \hat{t}) .
$$

We thus have

$$
\forall \hat{s} \in \mathbb{R}, \eta \in A, k \in \mathbb{N}_{0}: \frac{\mathrm{d}^{k}}{\mathrm{~d} \hat{s}^{k}} f(\hat{s}, \tan \eta \hat{s})=0 .
$$

Using induction over $k$, we can rewrite (24) in terms of the higher order partial derivatives $f_{m, n}$ to obtain:

$$
\forall k \in \mathbb{N}_{0}, \hat{s} \in \mathbb{R}, \eta \in A: \quad \sum_{i=0}^{k}(\tan \eta)^{i}\left(\begin{array}{c}
k \\
i
\end{array}\right) f_{k-i, i}(\hat{s}, \tan \eta \hat{s})=0 .
$$

For $\hat{s}=0$, the left side of this equation is a polynomial in $\tan \eta$. Because $\{\tan \eta \mid \eta \in A\}$ is infinite, the polynomial has infinitely many zeros and is therefore identically zero; i.e., we have

$$
\forall m, n \in \mathbb{N}_{0}: f_{m, n}(0,0)=0 .
$$


Because the characteristic function $\Phi^{\eta}$ satisfies (21) it is straightforward to verify that

$$
\forall s, t, \eta \in \mathbb{R}: \frac{\partial}{\partial \eta} \Phi^{\eta}(s, t)=f(\hat{s}(s, t, \eta), \hat{t}(s, t, \eta)),
$$

where

$$
\hat{s}(s, t, \eta)=s \cos \eta-t \sin \eta, \quad \hat{t}(s, t, \eta)=s \sin \eta+t \cos \eta .
$$

The chain rule and induction over $m+n$ imply that

$$
\frac{\partial^{m}}{\partial s^{m}} \frac{\partial^{n}}{\partial t^{n}} f(\hat{s}(s, t, \eta), \hat{t}(s, t, \eta))
$$

is a linear combination of

$$
\left\{f_{k, l}(\hat{s}(s, t, \eta), \hat{t}(s, t, \eta)) \mid k+l \leq m+n\right\} .
$$

This together with $(\hat{s}(0,0, \eta), \hat{t}(0,0, \eta))=(0,0)$ and $(25)$ implies that

$$
\left.\frac{\partial^{m}}{\partial s^{m}} \frac{\partial^{n}}{\partial t^{n}} f(\hat{s}(s, t, \eta), \hat{t}(s, t, \eta))\right|_{(s, t, \eta)=(0,0, \eta)}=0 .
$$

From (26) and (27) we get

$$
\forall m, n \in \mathbb{N}_{0}, \eta \in \mathbb{R}: \frac{\partial}{\partial \eta} \Phi_{m, n}^{\eta}(0,0)=0,
$$

which implies (22).

\section{Appendix B: Correlation}

Let $\sigma_{u v}=\operatorname{Cov}[\tilde{u}, \tilde{v}] \neq 0$. We exclude the trivial special case of perfect correlation; i.e., we assume that $\left|\sigma_{u v}\right|<\sigma_{u} \sigma_{v}$.

Proposition 1 remains true, albeit with different values for $\alpha_{N}, \beta_{N}, \lambda_{N}$ and $\mu_{N}$. To see this, note that (14) still holds, hence we can still conclude that $E[\tilde{u} \mid \tilde{v}]$ is an affine function of $\tilde{v}$, implying

$$
E[\tilde{u} \mid \tilde{v}]=\frac{\sigma_{u v}}{\sigma_{v}^{2}}(\tilde{v}-E[\tilde{v}])+E[\tilde{u}] .
$$

Now the same steps as in the proof of Proposition 1 can be used to compute the parameter values $\alpha_{N}, \beta_{N}, \lambda_{N}$ and $\mu_{N}$. In particular, one finds that

$$
\lambda_{N}=\frac{-(N-1) \sigma_{u v}+\sqrt{(N-1)^{2} \sigma_{u v}^{2}+4\left(\frac{\sigma_{u v}^{2}}{\sigma_{v}^{2}}+(N+1)^{2} \sigma_{u}^{2}\left(1-\frac{\sigma_{u v}^{2}}{\sigma_{u}^{2} \sigma_{v}^{2}}\right)\right) N \sigma_{v}^{2}}}{2\left(\frac{\sigma_{u v}^{2}}{\sigma_{v}^{2}}+(N+1)^{2} \sigma_{u}^{2}\left(1-\frac{\sigma_{u v}^{2}}{\sigma_{u}^{2} \sigma_{v}^{2}}\right)\right)} .
$$


One then works with the normalized random variable $\tilde{v}^{*}$ and the normalized uncorrelated part of $\tilde{u}^{*}$,

$$
\tilde{z}^{*}=\frac{1}{\sqrt{1-\frac{\sigma_{u v}^{2}}{\sigma_{u}^{2} \sigma_{v}^{2}}}}\left(\tilde{u}^{*}-\frac{\sigma_{u v}}{\sigma_{u} \sigma_{v}} \tilde{v}^{*}\right)
$$

to find the following necessary and sufficient condition parallel to (10) for the existence of a linear equilibrium for all $N$ :

$E\left[\frac{-\gamma_{N}}{\sqrt{\gamma_{N}^{2}+\delta_{N}^{2}}} \tilde{v}^{*}+\frac{\delta_{N}}{\sqrt{\gamma_{N}^{2}+\delta_{N}^{2}}} \tilde{z}^{*} \mid \frac{\delta_{N}}{\sqrt{\gamma_{N}^{2}+\delta_{N}^{2}}} \tilde{v}^{*}+\frac{\gamma_{N}}{\sqrt{\gamma_{N}^{2}+\delta_{N}^{2}}} \tilde{z}^{*}\right]=0 \forall N \in \mathbb{N}$,

where

$$
\gamma_{N}=\sigma_{v}-\lambda_{N} \frac{\sigma_{u v}}{\sigma_{v}}, \quad \delta_{N}=\lambda_{N}(N+1) \sigma_{u} \sqrt{1-\frac{\sigma_{u v}^{2}}{\sigma_{u}^{2} \sigma_{v}^{2}}} .
$$

Once one has shown that the set of angles replacing $A$,

$$
A^{\prime}=\left\{\eta \in(0,2 \pi) \mid \exists N \in \mathbb{N}: \frac{\gamma_{N}}{\sqrt{\gamma_{N}^{2}+\delta_{N}^{2}}}=\sin \eta\right\}
$$

is infinite, the remaining analysis is parallel to the uncorrelated case: it follows that the moments of $\left(\tilde{v}^{*}, \tilde{z}^{*}\right)$ are rotation invariant, hence $(\tilde{u}, \tilde{v})$ is elliptic if it is determined by its moments.

To show that $A^{\prime}$ is infinite, it is sufficient to prove the following two claims:

$$
\begin{aligned}
& \gamma_{N}>0 \text { for all sufficiently large } N, \\
& \frac{\gamma_{N}}{\sqrt{\gamma_{N}^{2}+\delta_{N}^{2}}} \rightarrow 0 \text { as } N \rightarrow \infty
\end{aligned}
$$

A straightforward computation shows that, as $N \rightarrow \infty$,

$$
\lambda_{N} \sqrt{N} \rightarrow \frac{\sigma_{v}}{\sigma_{u} \sqrt{1-\frac{\sigma_{u v}^{2}}{\sigma_{u}^{2} \sigma_{v}^{2}}}}>0
$$

Hence, $\gamma_{N} \rightarrow \sigma_{v}$ and $\delta_{N} \rightarrow \infty$, from which (28) and (29) are immediate. 


\section{References}

[1] Bagnoli, M., Viswanathan, S., and Holden, C.: On the existence of linear equilibria in models of market making, Mathematical Finance 11, 1-31 (2001)

[2] Berg, C. : Recent results about moment problems, in: Probability Measures on Groups and Related Structures, XI Proceedings Oberwolfach 1994, ed. Heyer, H., Singapore: World Scientific 1995

[3] Berg, C., Thill, M.: Rotation invariant moment problems, Acta Mathematica 167, 207-227 (1991)

[4] Bélisle, C., Massé, J.-C., Ransford, T.: When is a probability measure determined by infinitely many projections?, The Annals of Probability 25, 767-786 (1997)

[5] Billingsley, P.: Probability and Measure, Third Edition, New York: Wiley 1995.

[6] Chamberlain, G.: A characterization of the distributions that imply mean-variance utility functions, Journal of Economic Theory 29, 185$201(1983)$

[7] Cramér, H., Wold, H.: Some theorems on distribution functions, Journal of the London Mathematical Society 11, 290-294 (1936).

[8] Fang, K.-T., Kotz, S., and Ng, K.-W.: Symmetric multivariate and related distributions. New York: Chapman and Hill 1990

[9] Foster, F., Viswanathan, S.: The effect of public information and competition on trading volume and price volatility, Review of Financial Studies 6, 23-56 (1993)

[10] Gilbert, W.M.: Projections of probability distributions, Acta Math. Acad. Sci. Hungar. 6, 195-198 (1955)

[11] Hardin, C.: On the Linearity of Regression, Zeitschrift für Wahrscheinlichkeitstheorie und verwandte Gebiete 61, 291-302 (1982)

[12] Kyle, A.: Continuous auctions and insider trading, Econometrica 53, 1315-1335 (1985)

[13] Lukacs, E., Laha, R.: Applications of characteristic functions. London: Griffin 1969 
[14] Nöldeke, G., Tröger, T.: Existence of linear equilibria in the Kyle model with multiple informed traders, Economics Letters 72, 159-164 (2001)

[15] Nöldeke, G., Tröger, T.: On the existence of linear equilibria in the Rochet-Vila model of market making, Bonn Econ Discussion paper $19 / 2004$ (2004)

[16] O'Hara, M.: Market microstructure theory. Cambridge, Mass.: Basil Blackwell 1995

[17] Peterson, L.C.: On the relation between the multidimensional moment problem and the one-dimensional moment problem, Mathematica Scandinavica 51, 361-366 (1982)

[18] Rochet, J.-C., Vila, J.-L.: Insider trading without normality, Review of Economic Studies 61, 131-152 (1994)

[19] Shohat, J.A., Tamarkin, J.D.: The problem of moments. New York: Amer. Math. Soc. Colloquium Publ. 1950 